\title{
Complete State-Wide Outcomes in Elective Colon Cancer Surgery
}

Ian G Faragher, FCSSANZ, FRACS ${ }^{a, b}$

Michael K-Y Hong, DMedSc, FRACS ${ }^{a}$

Douglas Stupart, FRACS ${ }^{\mathrm{C}}$

David A Watters, FRACS

Justin Yeung, DM, FRCS (General Surgery) ${ }^{a, b}$

${ }^{a}$ Colorectal Surgery Unit, Western Health, Melbourne, Australia

${ }^{\mathrm{b}}$ Academic Department of Surgery, Western Health, Melbourne, Australia

'Department of Surgery, The Geelong Hospital, Deakin University, Geelong, Victoria, Australia

Colorectal Surgery Unit, Western Health, Melbourne, Australia

Short title: Outcomes in Colon Cancer Surgery

Conflicts of Interest: none

Figures: 3

Tables: 1

This is the author manuscript accepted for publication and has undergone full peer review but has not been through the copyediting, typesetting, pagination and proofreading process, which may lead to differences between this version and the Version of Record. Please cite this article as doi: $10.1111 /$ ans.14872

This article is protected by copyright. All rights reserved. 
Supplementary Tables: 1

Word Count (abstract): 250

Word Count (manuscript): 1835

Corresponding Author:

Ian G Faragher

Colorectal and General Surgery Unit

E: ian.faragher@wh.org.au

This paper was presented in part at the GSA Annual Scientific Meeting, Melbourne, $30^{\text {th }}$ September 2016. 


\section{Abstract}

Introduction: Maintaining high standards in colon cancer surgery requires the measurement of quality indicators and the re-allocation of resources to address deficiencies. We used state-wide administrative data to determine the inpatient mortality for patients undergoing elective colon cancer surgery and to compare individual hospital rates.

Methods: The Dr Foster Quality Investigator Tool was used to explore the Victorian Admitted Episodes Dataset for elective admissions for colon cancer surgery between 2012 and 2016. The inpatient mortality rate, 30-day readmission rate and the proportion of patients with increased length of stay were measured. Risk-adjusted rates were used to compare public and private hospitals. A peer group of 14 hospitals were studied using funnel plots to determine inter-hospital variation in mortality.

Results: There were 6120 colectomies performed for colon cancer in Victoria over three years. The crude inpatient mortality rate was $1.3 \%$. It was significantly higher in public than private hospitals, even after risk adjustment. Variation in crude mortality was demonstrated among 14 
select hospitals. The lowest volume hospitals had significantly higher inpatient mortality rates. Right hemicolectomy was the commonest procedure performed.

Conclusion: Using an efficient method of complete state-wide data capture, we have demonstrated that the inpatient mortality rate after elective colon cancer surgery in Victoria is low. However, complexity remains around the interpretation of inter-hospital variation, defining outliers, and comparing outcomes between public and private hospitals. Resolving these complexities and defining additional quality indicators remain a priority in the use of administrative data to audit the quality of colon cancer care.

Key Words: cancer, colectomy, outcomes, administrative data, quality indicator

\section{Introduction}

The optimal method for measuring and benchmarking quality in colorectal cancer surgery in Australia remains controversial. Reporting of outcomes from colorectal cancer surgery have previously relied on institution-based reporting or databases reliant on self-reporting. The Bi-

This article is protected by copyright. All rights reserved. 
National Colorectal Cancer Audit (BCCA) recently published on the inpatient mortality rate from participants across Australia and New Zealand[1]. State-wide outcomes from the Queensland Colorectal Cancer Audit Report were also recently published[2].

The BCCA, whilst providing comprehensive outcomes from participants, relies on voluntary self-reporting. In 2017 , around $13 \%$ of new colorectal cancer cases from Australia and New Zealand were included[3]. On the contrary, the Queensland Audit used administrative data linked with other clinical databases to provide complete state-wide data. The routine nature of administrative data and their ability to provide information about every hospital admission makes them a valuable source of complete data capture[4]. Therefore, administrative data are increasingly used for outcomes research and hospital performance benchmarking $[5,6]$.

A key challenge in the performance benchmarking process is the identification of quality indicators. In the case of administrative data, it is not a question of what parameters to collect, but rather what has been collected. Inpatient and 30-day mortality rates are commonly used quality indicators for hospital benchmarking and comparing cancer surgery outcomes[7-10].

This article is protected by copyright. All rights reserved. 
We aimed to determine the inpatient mortality rate for elective colon cancer surgery using a state-wide administrative database. Readmission rates and prolonged length of stay (LOS) were other parameters explored. A peer group of hospitals was studied to explore individual hospital variation in inpatient mortality.

\section{Methods}

The Victorian Admitted Episodes Dataset (VAED) was examined for all elective admissions for colon cancer surgery between $1^{\text {st }}$ July 2012 and $30^{\text {th }}$ June 2016. This cohort was defined using diagnostic codes from the International Classification of Diseases $10^{\text {th }}$ Revision Australian Modification (ICD-10-AM) and procedure codes from the Australian Classification of Health Interventions (ACHI). These codes included colonic cancer diagnostic codes and colectomy procedure codes (Supplementary Table 1).

Procedure codes for colonic resections, including Hartmann's procedure and high anterior resection, were combined only with diagnostic codes for sigmoid and rectosigmoid cancer to ensure that only colonic cancer cases were included. The frequency of use for every procedure

This article is protected by copyright. All rights reserved. 
code was recorded. Specific codes for laparoscopic colectomies were only used from 2013 onwards, so meaningful comparison of laparoscopic with open colectomy outcomes was not possible. Furthermore, there is no specific code for laparoscopic high anterior resection.

The Dr Foster Quality Investigator Tool was used to interrogate the VAED. This tool was provided by Dr Foster (Telstra Health). It is designed to assist healthcare organisations to improve clinical quality and patient safety through measurement, comparison and benchmarking of administrative data.

The primary outcome measured was crude inpatient mortality rate. No data linkage was available to determine 30-day mortality. Readmissions within 30 days were defined as any admission, to any hospital in Victoria, within 30 days of discharge. Readmissions were allocated to the hospital performing the primary surgery. Prolonged LOS was defined as the upper quartile for the LOS. Within the defined cohort, outcomes were measured for public and private hospitals. Fourteen of a possible 22 public health services gave permission to be part of a peer group for comparison of individual hospital outcomes. 
Risk adjustment was performed using logistic regression to allow fairer comparison between various cohorts within the VAED for mortality. Factors used in the adjustment included age, gender, Charlson comorbidity score, admission source, number of emergency admissions in prior 12 months, patients under palliative care, and month and year of admission. The benchmark year used for risk modelling was July 2013 to June 2014.

Statistical analysis was performed within the Dr Foster Quality Investigator Tool. Chi-squared tests were used for proportions. Differences in comparisons were considered significant where the probability was less than 0.05 . A funnel plot was used to explore interhospital variation within the peer group of hospitals for inpatient mortality rate. Confidence levels of $95 \%$ and $99.8 \%$ were highlighted in this plot. Outliers were defined as those outside the $95 \%$ confidence level. This project was endorsed by the Dr Foster Intelligence Clinical Focus Group steering committee and approved by the Office of Research, Western Health (QA2017.46). 


\section{Results}

From July 2012 to June 2016 there were 6120 elective colectomies performed for colon cancer in Victoria (Table 1). The mean age of the cohort was 70 years with over two thirds of patients undergoing colectomy above the age of 65 years. These demographics were similar between the public and private hospitals, in which half the colectomies occurred in each. The peer group accounted for most of the colectomies performed in the public hospitals.

The overall crude inpatient mortality rate in Victoria was $1.3 \%$ (Figure 1). The crude rate was $1.7 \%$ in public hospitals, which was significantly higher than the $0.8 \%$ observed in private hospitals $(p<0.01)$. This significance held true after risk adjustment. This risk adjustment model achieved a c-statistic of 0.91 , indicating an excellent fit for the data. The 14-hospitals forming the peer group had a crude mortality rate of $1.8 \%$, and there was variation among the individual hospitals (Figure 2). The two hospitals with the lowest number of colectomies had crude mortality rates above the $95 \%$ confidence level but below the $99.8 \%$ level. These outliers remained after risk adjustment.

This article is protected by copyright. All rights reserved. 
The overall 30-day readmission rate was 6.8\%: higher in public hospitals (9.4\%) and lower in private hospitals (4.1\%). This difference was statistically significant $(p<0.01)$, even after risk adjustment. However, a c-statistic of 0.63 indicated a poor fit for the data. The lower readmission rate in private hospitals was offset by a higher proportion of patients with prolonged LOS (34\% compared with $29 \%$, p<0.01). The readmission rate was highest in the peer group, where the proportion of prolonged inpatient stay was lowest.

The most common elective colectomy performed in both the public and private hospitals was right hemicolectomy, followed by high anterior resection for colonic cancer (Figure 3). Excluding high anterior resection from analysis, the rate of laparoscopic surgery was at least $39 \%$ overall. The overall use of stoma was low at $4 \%$.

\section{Discussion}

A state-wide administrative dataset has been used to capture all elective colectomies for colon cancer in the state of Victoria. The crude inpatient mortality rate was $1.3 \%$ after elective colon cancer surgery across all hospitals in the state of Victoria between 2012 and 2016 . The mortality was significantly higher in public hospitals compared with private hospitals, even after risk adjustment. Analysis of a select peer 
group of 14 public Victorian hospitals demonstrated variation in inpatient mortality, with some low volume centres exceeding the $95 \%$ confidence level.

In comparison, the Queensland Colorectal Cancer Audit reported a crude inpatient mortality rate of $1.8 \%$ in 2016 , but those data also included surgery after rectal cancer[2]. We chose to separately analyse colon cancer to reduce the heterogeneity of our population. Similarly, the BCCA found the inpatient mortality rate to be $1.1 \%$ from over 10000 colorectal cancer cases[1]. This was across many hospitals from Australia and New Zealand, but they were self-reported cases without complete data capture. The Concord Hospital found their 30-day mortality to be $1.3 \%$ over three years, having declined from $4.6 \%$ in the 1970 s[11]. The National Bowel Cancer Audit based in England and Wales found their rate of 90-day mortality to be around 2\% after elective resection in 2014-15[12].

A US study using administrative data from the University Health System Consortium (UHC) database found an inpatient mortality rate of 1.6\% after elective colectomy[13]. They analysed 85260 patients from 195 hospitals from 2005 to 2011, but also included benign disease. They rejected the use of inpatient mortality as a quality parameter on the basis of a low event rate and narrow distribution width after risk adjustment. However, a few hospitals were found to have a mortality rate that exceeded their $95 \%$ confidence level, which is similar to our 
study. We would recommend that mortality rates, whilst not the only quality measure of value, certainly need to continue to be reported from national and state quality databases at the hospital level.

There were a significant number of elective colectomies performed for colon cancer in private hospitals. This has implications for surgical training as well as the governance of processes such as multidisciplinary meetings. The variety of colectomies was similar between public and private hospitals. The inpatient mortality and readmission rates were significantly lower in the private hospitals compared with the public hospitals. Health insurance status is a marker of other factors that may influence mortality rate, such as literacy, socioeconomic status and patient motivation[14]. These factors are difficult to risk adjust. A large study from the US found differences in mortality risk and complications between the insured and uninsured patients in the same hospitals even after controlling for comorbidities[15]. Therefore, comparison of health outcomes based on insurance status is a poor surrogate for the quality of healthcare provision.

Our study found statistical outliers in inpatient mortality among low volume centres, which raises several issues. Firstly, we defined outliers as being outside the $95 \%$ confidence interval. If 20 hospitals were compared, the mortality rate for one hospital would be expected to fall outside this interval. A low event rate for mortality means that low volume centres are heavily affected by each inpatient death. Other 
studies have shown only a weak association between avoidable deaths and standardised mortality[16]. Coupled with a low event rate, inpatient mortality may not reflect the quality of hospital care[17]. In Victoria, low volume centres tend to be smaller regional hospitals that serve more distant communities. Perhaps it is more important for low volume centres to monitor their colon cancer outcomes than high volume centres. Centralisation of services may not be the solution, and additional resources should be allocated to reviewing and improving the quality of care where possible.

Whilst successfully measured, readmission rates and prolonged LOS were difficult to compare meaningfully, and as such are also difficult to use as indicators of quality. Our study shows that a 30-day readmission rate after elective colon cancer surgery is inevitable at a rate of 7 to 14\%. In the context of private health insurance companies introducing penalties for complications[18] and readmissions, complete elimination of readmissions is probably aspirational rather than realistic, and could create a perverse incentive to not readmit when that would compromise patient safety. For administrative data to be useful for hospital benchmarking in colon cancer surgery, additional quality indicators are needed. 
The most significant limitation in our study was the lack of cancer staging information. This limits the effectiveness of risk adjustment methods in providing fair comparisons between patient cohorts. A lack of opportunity to explore other potential quality indicators, such as unplanned return to theatre which may inversely correlate with mortality, stemmed from an inability to define suitable endpoints from within the administrative dataset. This could be improved by linking administrative data with other datasets or adding quality data fields.

\section{Conclusion}

Through the use of an administrative dataset not reliant on self-reporting, we have achieved complete state-wide data capture for outcomes in elective colon cancer surgery. In Victoria, in-hospital mortality is low, but inter-hospital variation exists. Lower volume hospitals have a higher mortality rate, but the use of mortality for hospital benchmarking in colon cancer surgery remains debatable. 


\section{Acknowledgements}

The authors would like to thank Chris Brown from Dr Foster, Telstra Health for his work in the interrogation of the VAED and providing statistical support. This project was supported by the Department of Health and Human Services, Victoria.

\section{References}

1. Teloken, P.E., et al., Analysis of mortality in colorectal surgery in the Bi-National Colorectal Cancer Audit. ANZ J Surg, 2016. 86(6): p. 4548.

2. Government, Q. Queensland Colorectal Cancer Audit Report 2016 [cited 2017 12 February]; Available from: https://qccat.health.qld.gov.au/documents/Colorectal Qld.pdf.

3. Heriot, A., et al., The Bi-National Colorectal Cancer Audit Report 2018. 2018.

4. Hong, M.K., et al., Operative intervention rates for acute diverticulitis: a multicentre state-wide study. ANZ J Surg, 2015. 85(10): p. 7348.

5. Aylin, P., A. Bottle, and A. Majeed, Use of administrative data or clinical databases as predictors of risk of death in hospital: comparison of models. BMJ, 2007. 334(7602): p. 1044. 
6. Bottle, A., et al., Global comparators project: international comparison of hospital outcomes using administrative data. Health Serv Res, 2013. 48(6 Pt 1): p. 2081-100.

7. Morris, E.J., et al., Thirty-day postoperative mortality after colorectal cancer surgery in England. Gut, 2011. 60(6): p. 806-13.

8. Osler, M., et al., Hospital variation in 30-day mortality after colorectal cancer surgery in denmark: the contribution of hospital volume and patient characteristics. Ann Surg, 2011. 253(4): p. 733-8.

9. Panis, Y., et al., Mortality after colorectal cancer surgery: a French survey of more than 84,000 patients. Ann Surg, 2011. 254(5): p. 73843; discussion 743-4.

10. Cone, M.M., et al., Dramatic decreases in mortality from laparoscopic colon resections based on data from the Nationwide Inpatient Sample. Arch Surg, 2011. 146(5): p. 594-9.

11. Dent, O.F., et al., Trends in short-term outcomes after resection of colorectal cancer: 1971-2013. ANZ J Surg, 2017. 87(1-2): p. 39-43.

12. Ireland, T.A.o.C.o.G.B.a. National Bowel Cancer Audit. [cited 2018; Available from: https://www.acpgbi.org.uk/research/bowel-cancer/.

13. Billeter, A.T., et al., Mortality after elective colon resection: the search for outcomes that define quality in surgical practice. J Am Coll Surg, 2012. 214(4): p. 436-43; discussion 443-4.

14. Akinyemiju, T., Q. Meng, and N. Vin-Raviv, Race/ethnicity and socio-economic differences in colorectal cancer surgery outcomes: analysis of the nationwide inpatient sample. BMC Cancer, 2016. 16: p. 715.

15. Kelz, R.R., et al., Morbidity and mortality of colorectal carcinoma surgery differs by insurance status. Cancer, 2004. 101(10): p. 2187-94.

16. Doran, T., K. Bloor, and A. Maynard, The death of death rates? BMJ, 2015. 351: p. h3466.

17. Hogan, H., et al., Avoidability of hospital deaths and association with hospital-wide mortality ratios: retrospective case record review and regression analysis. BMJ, 2015. 351: p. h3239.

This article is protected by copyright. All rights reserved. 
18. Davis, C., Non-reimbursement for preventable health care-acquired conditions. Med J Aust, 2016. 205(1): p. 42-3. 


\section{Figure 1}

Crude inpatient mortality rates with $95 \%$ confidence intervals, together with corresponding resection volumes in Victoria.

\section{Figure 2}

Funnel plot showing the variation in inpatient mortality for the 14 individual hospitals within the select peer group.

\section{Figure 3}

Frequency of procedure types divided into public and private, July 2012 to June 2016.

Table 1

Summary of crude outcomes for elective cancer resections in Victoria, July 2012 to June 2016. 
This article is protected by copyright. All rights reserved. 


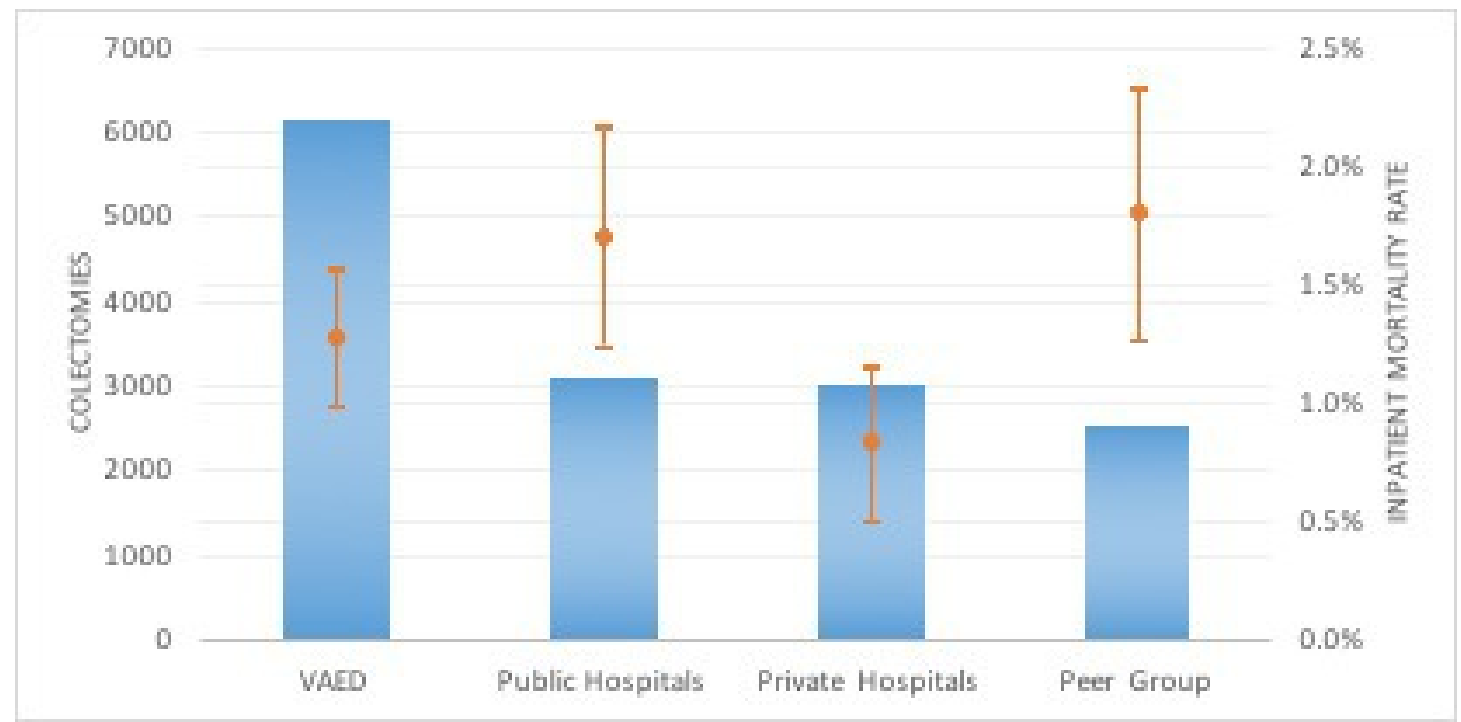

Figure 1.jpg

This article is protected by copyright. All rights reserved. 


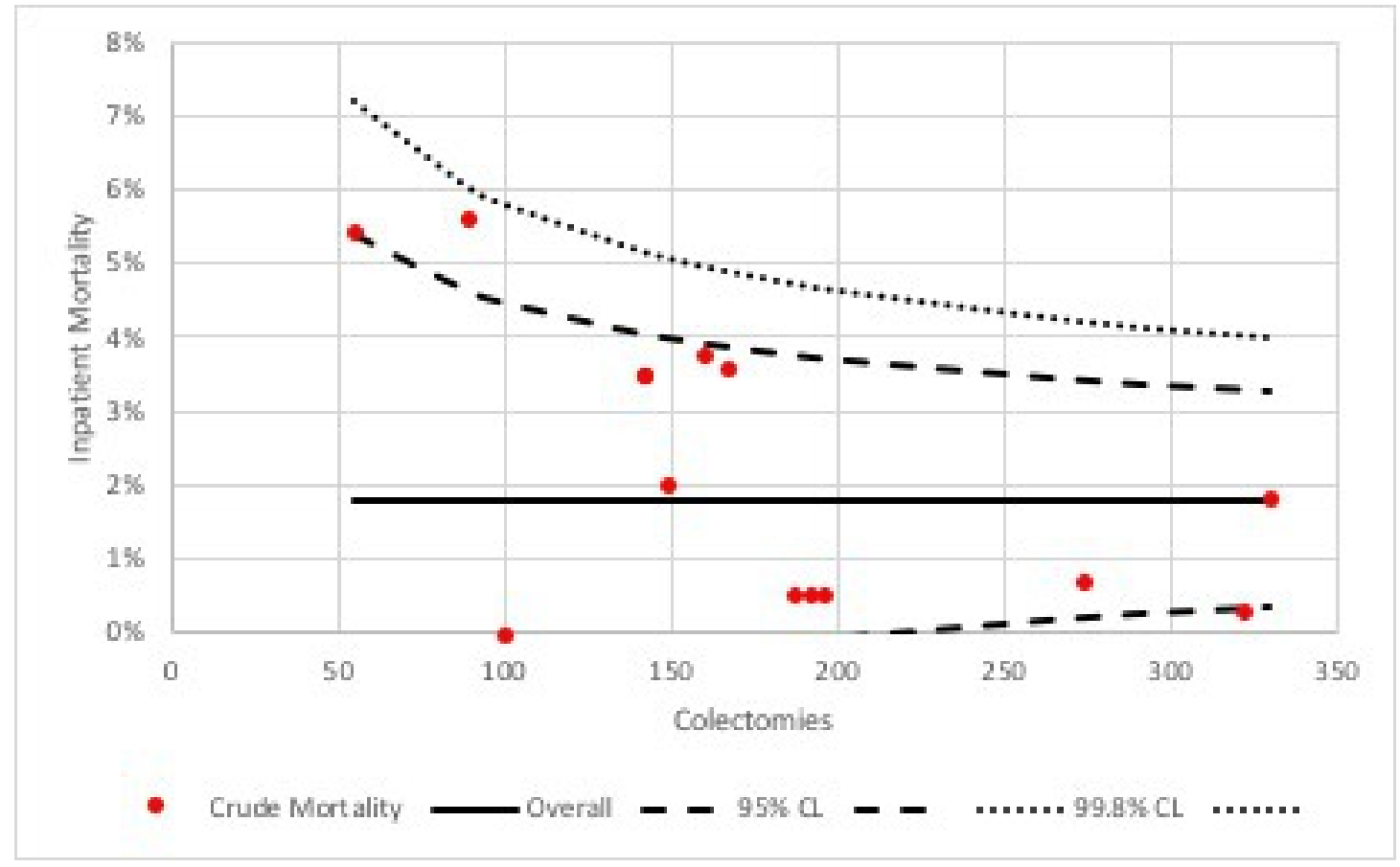

Figure 2.jpg

This article is protected by copyright. All rights reserved. 


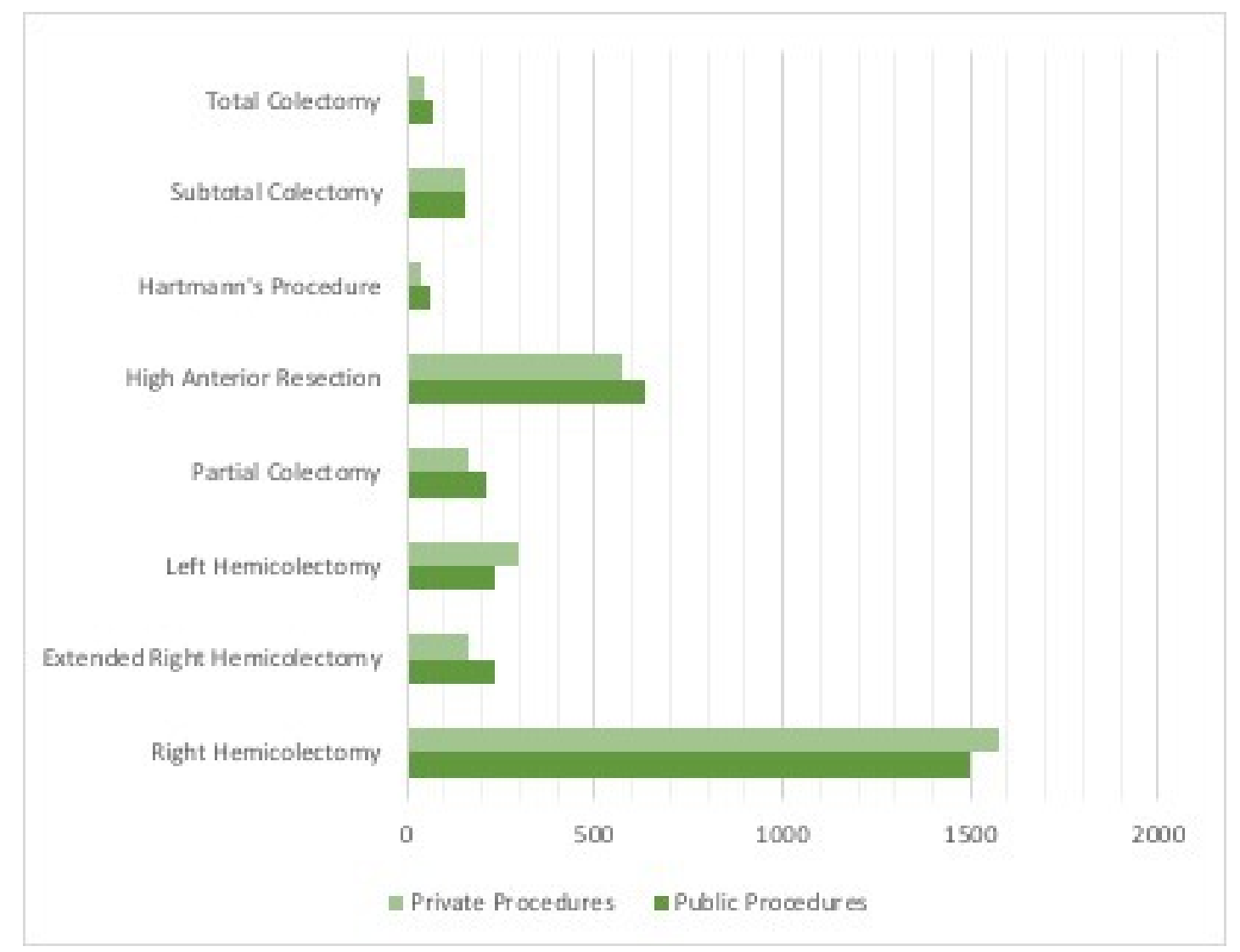

Figure 3.jpg

This article is protected by copyright. All rights reserved. 


\section{Table 1}

Summary of crude outcomes for elective cancer resections in Victoria, July 2012 to

June 2016.

\begin{tabular}{|c|c|c|c|c|c|c|c|c|}
\hline Cohort & Colectomies & Mean Age ( \pm sd) & $\%$ Male & $\begin{array}{c}\text { Median } \\
\text { LOS [range } \\
\text { of medians] }\end{array}$ & $\begin{array}{l}\text { Inpatient } \\
\text { Mortality }\end{array}$ & $\begin{array}{c}\text { Relative Risk of } \\
\text { Mortality }\end{array}$ & Prolonged LOS & 30-Day Readmissions \\
\hline Public Hospitals & 3105 & $69 \pm 13$ & $64 \%$ & $8[6-10]$ & $53(1.7 \%)$ & $0.58(0.43-0.75)$ & $29 \%$ & 291 (9.4\%) \\
\hline Private Hospitals & 3015 & $71 \pm 13$ & $52 \%$ & $8[2-11]$ & $25(0.8 \%)^{*}$ & $0.28(0.18-0.42)$ & $34 \%$ & $125(4.1 \%)^{*}$ \\
\hline
\end{tabular}

$$
*_{p}<.01
$$




\section{University Library}

\section{- M M N E R VA A gateway to Melbourne's research publications}

Minerva Access is the Institutional Repository of The University of Melbourne

Author/s:

Faragher, IG;Hong, MK-Y;Stupart, D;Watters, DA;Yeung, J

Title:

Complete state-wide outcomes in elective colon cancer surgery

Date:

2018-11-01

Citation:

Faragher, I. G., Hong, M. K. -Y., Stupart, D., Watters, D. A. \& Yeung, J. (2018). Complete state-wide outcomes in elective colon cancer surgery. ANZ JOURNAL OF SURGERY, 88 (11), pp.1174-1177. https://doi.org/10.1111/ans.14872.

Persistent Link:

http://hdl.handle.net/11343/284655 